\title{
Review: continuing antidepressants for 12 months following acute treatment may reduce the risk of relapse in depression
}

Geddes JR, Carney SM, Davies C et al. Relapse prevention with antidepressant drug treatment in depressive disorders: a systematic review. Lancet 2003; Feb, 361:653-61.

\section{QUESTION: In people with depressive disorders, how long should antidepressant treatment be continued to prevent relapse?}

\section{Design}

Systematic review with meta-analysis.

\section{Data sources}

The authors searched the Cochrane Collaboration Depression, Anxiety, and Neurosis Controlled Trials Register (CCDANCTR). This incorporates searches of Medline (1966-April 2000); Embase (1980-April 2000); CINAHL (1982-April 2000); PsycLit (1974-April 2000); Psyndex (1977-December 1999), and LILACS (1982December 1999). The reviewers screened the reference lists of all selected studies, other relevant papers and major textbooks, and contacted the authors of identified papers, experts in the field, and manufacturers of antidepressants for additional studies.

\section{Study selection}

Published and unpublished randomised trials were eligible if they compared continuing with discontinuing antidepressant treatment in people with depressive disorders who responded to acute treatment. The authors included 31 trials with 4410 participants. Most trials were of 12 months duration.

\section{Data extraction}

Two reviewers extracted data about participant, intervention, and outcome characteristics. Data were checked by a third reviewer.

\section{Main results}

The average rate of relapse was $41 \%$ in people receiving placebo compared with $18 \%$ for those continuing antidepressant treatment. Continuing treatment reduced the likelihood of relapse by $70 \%$ (95\% CI $62 \%$ to $78 \%, \mathrm{p}<0.00001)$. The reduced risk of relapse appeared largely independent of the initial risk of relapse, duration of previous treatment, and the duration of randomly allocated therapy. A greater number of participants allocated antidepressants withdrew from the trials compared to placebo (18\% v $15 \%$ respectively, odds ratio $1.30,95 \%$ CI 1.07 to 1.59 ). Evidence about the effects of continuing antidepressant treatment for more than one year was limited.

\section{Conclusions}

Continuing antidepressants for 12 months after acute treatment may benefit many people with recurrent depressive disorder. Further trials are needed to establish the optimum length of therapy, especially for those at low risk of relapse.

\section{COMMENTARY}

Concerns about the premature discontinuation of antidepressant treatment in primary care were first expressed 30 years ago.' In the absence of long term data from randomised trials, it has been assumed that early cessation of therapy contributes to the preventable morbidity associated with depression. ${ }^{2}$ This landmark review by Geddes et al addresses one of the most important clinical dilemmas in the management of depression: how long should antidepressant treatment continue in order to minimise the risk of subsequent relapse?

We do not have a definitive answer to this question, primarily due to the limited data available, most of which come from studies conducted mainly in secondary care settings with patients at high risk of relapse. Despite the limitations inherent in meta-analysis (differences between studies in patients recruited, antidepressants used, length of antidepressant treatment before randomisation, and whether results are reported on an intention to treat basis), this review suggests that an additional year of antidepressant treatment is likely to reduce the risk of relapse by about half in people at high risk, irrespective of which class of antidepressant is used or the length of prior treatment.

For people at lower risk of relapse, the benefit of continuing treatment is less clear. In terms of secondary prevention, this is a key patient group. It is here that the best opportunities lie to prevent development of recurrent depression, where the burden of illness becomes particularly high. These patients tend to be managed in primary care settings. Although available data are fewer for this group, Geddes et al suggest that a continuation of therapy may still be beneficial, though there is a need for prospective randomised studies to confirm these tentative findings.

MRPharm S John Donoghue, BSc School of Pharmacy and Chemistry Liverpool John Moores University United Kingdom
For correspondence: $J$ Geddes, University of Oxford, Department of Psychiatry, Warneford Hospital, Oxford, UK. john.geddes@ psych.ox.ac.uk
1 Johnson DAW. Treatment of depression in general practice. BMJ 1973; 1: 18-20.

2 Donoghue JM, Taylor DM. Suboptimal use of antidepressants in the treatment of depression. CNS Drugs 2000; 13: 365-83. 